\title{
Feasibility study of hand-carried ultrasound-guided retrievable inferior vena cava filter placement
}

\author{
Hang Zhu ${ }^{1 \#}$, Wen-Juan Du ${ }^{2 \#}$, Xiao-Hua Wang ${ }^{3 \#}$, Yang Yang ${ }^{4}$, Dekyi $^{5}$, Yun-Dai Chen ${ }^{1}$, Jing Zhao ${ }^{6}$ \\ ${ }^{1}$ Department of Cardiovascular Medicine, Chinese PLA General Hospital, Beijing, China; ${ }^{2}$ Disciplinary Degree Office, Graduate School of Chinese \\ PLA General Hospital, Beijing, China; ${ }^{3}$ Department of Nephrology, Second Medical Center, Chinese PLA General Hospital, Beijing, China; \\ ${ }^{4}$ Department of Clinical Medicine, The First Clinical College of Hainan Medical University, Haikou, China; ${ }^{5}$ Department of Cardiovascular \\ Medicine at High Altitude, People's Hospital of Tibet Autonomous Region, Lasa, China; ${ }^{6}$ Scientific Research Office, Department of Medical \\ Services, Chinese PLA General Hospital, Beijing, China \\ Contributions: (I) Conception and design: H Zhu; (II) Administrative support: J Zhao; (III) Provision of study materials or patients: Y Yang, YD Chen; \\ (IV) Collection and assembly of data: XH Wang, Dekyi; (V) Data analysis and interpretation: WJ Du; (VI) Manuscript writing: All authors; (VII) \\ Final approval of manuscript: All authors. \\ "These authors contributed equally to this work. \\ Correspondence to: Dr. Yun-Dai Chen. Department of Cardiovascular Medicine, Chinese PLA General Hospital, No. 28, Fuxing Road, Haidian \\ District, Beijing 100853, China. Email: ydc301@163.com; Dr. Jing Zhao. Scientific Research Office, Department of Medical Services, Chinese PLA \\ General Hospital, No. 28, Fuxing Road, Haidian District, Beijing 100853, China. Email: jingzhao_cpgh@163.com.
}

\begin{abstract}
Background: With the development of ultrasonic diagnostic techniques in recent years, ultrasoundguided placement of inferior vena cava (IVC) filters has been widely used in clinics, and satisfactory results have been achieved. Our study aims to observe the accuracy of hand-carried ultrasound-guided retrievable vena cava filter placement, evaluate the feasibility and safety of this new method, and provide a scientific and effective interventional method and clinical data to prevent acute pulmonary embolism (PE) after battle injury of limbs.
\end{abstract}

Methods: Two hundred patients with post-traumatic thrombosis of the extremities were enrolled. The renal vein was located under the guidance of hand-carried ultrasound. The retrievable filter was fixed 1-2 $\mathrm{cm}$ below the opening of the renal vein. The self-expanding filter was used after the filter's position was confirmed by injecting the contrast agent under the digital subtraction angiography (DSA) fluoroscopy.

Results: All the 200 patients underwent the operation successfully. The position of the hand-carried ultrasound localizer was consistent with the DSA localizer. All the filters were expanded smoothly. No complications related to the operation occurred.

Conclusions: The study concluded that the hand-carried ultrasound-guided retrievable vena cava filter placement has a high success rate and can prevent acute PE after limbs' battle injury.

Keywords: Portable ultrasound; digital subtraction angiography (DSA); retrievable inferior vena cava filter

Submitted Feb 22, 2021. Accepted for publication Apr 13, 2021.

doi: $10.21037 /$ atm-21-1290

View this article at: http://dx.doi.org/10.21037/atm-21-1290

\section{Introduction}

Catastrophic earthquakes, highway accidents, and modern wars can produce many casualties in a short period and have a very high mortality rate, of which limb injury is the most common cause of death $(1,2)$. Trauma is a high-risk factor for thrombosis in the lower extremities, and studies have shown that traumatic thrombosis occurs in $2.5 \%$ to $55 \%$ (3). Pulmonary embolism (PE) is a common complication of thrombosis (4) and is the leading cause of death among the many injured (5). PE was found in $72 \%$ to $82 \%$ of patients with lower limb thrombosis, and PE was found in $34 \%$ to $58 \%$ (6) of patients with lower limb thrombosis. Inferior 
vena cava (IVC) is medical device that has been widely used an effective measure to prevent $\mathrm{PE}$ in the clinic in recent years. It has been proved to greatly reduce the morbidity and mortality of PE (7). For most patients with Limb Trauma, the risk of handling is significantly increased, says Stearley (8). Complications, including vital sign disorders and cardiac arrest, may occur during transport in $15.5 \%$ of patients (8). At present, we usually place IVC filters under the $\mathrm{X}$-ray location, but because of the huge equipment, expensive, and cannot meet the patients who cannot move the limbs of the need for diagnosis and treatment. With the development of ultrasonic diagnostic techniques, ultrasound-guided placement of IVC filters has been widely used in clinics, and satisfactory results have been achieved $(9,10)$. The appearance of a hand-carried ultrasonic diagnostic instrument provides the possibility to solve the limitation of the vena cava filter used in battlefield first aid, earthquake, and disaster rescue. In this study, we evaluated the safety and efficacy of a novel, hand-carried ultrasound diagnostic instrument, which was more portable and had higher resolutions compared to previous models, in retrievable vena cava filter placement. A total of 200 patients were admitted to our hospital and treated with a hand-carried ultrasound-guided retrievable vena cava filter after thrombosis injury. To explore the safety and feasibility of hand-carried ultrasound-guided retrievable vena cava filter placement to prevent acute PE after battle trauma of limbs.

We present the following article in accordance with the STROBE reporting checklist (available at http://dx.doi. org/10.21037/atm-21-1290).

\section{Methods}

\section{Study subjects}

From June 2018 to June 2020, 200 patients who were admitted to our hospital and the medical conjoined hospital, and underwent IVC filter implantation were enrolled. All patients were diagnosed with lower limb thrombosis (DVT) by ultrasonography, including 108 males and 92 females, aged 24 to 56 , with an average age of $41 \pm 3.24$. There were 68 cases of the left lower limb and 132 cases of the right lower limb.

Inclusion criteria: (I) there were contraindications to anticoagulant therapy or severe bleeding complications after anticoagulant therapy; (II) PE still occurs after anticoagulant therapy; (III) venous thrombectomy includes direct catheter thrombolysis and thrombolysis of the dorsalis pedis vein and via arterial route before thrombectomy; (IV) residual thrombosis after the first PE; (V) free and suspended massive thrombus were found in the deep venous system of the lower extremity; (VI) patients with or likely to have unilateral lower extremity thrombosis, including fractures of the lower extremities and severe trauma to the pelvis.

Exclusion criteria: (I) severely obese persons without access to renal vein openings and blood flow detected by portable ultrasound; (II) patients with severe renal dysfunction whose glomerular filtration rate $\leq 15 \mathrm{~mL} / \mathrm{min}$ or severe allergy cannot be treated with iodinated contrast medium; (III) X-ray intolerance in pregnant women.

All procedures performed in this study involving human participants were in accordance with the Declaration of Helsinki (as revised in 2013). The study was approved by ethics committee of Chinese PLA General Hospital and informed consent was taken from all the patients.

\section{Instruments and operation}

\section{Instruments}

GE VSCAN 2.0 Dual Probe portable color doppler ultrasound was used for ultrasound guidance. According to various parts of the body, the Probe frequency was 3-5 and 6-12 MHz. Digital subtraction angiography (DSA) was used for Siemens Angiostar digital subtraction angiography. Advanced Technology Aegisy VENA CAVA filter and delivery system, filter components are bracket, umbrella, connecting rod, and hook-shaped part.

\section{Operation}

IVC filter implantation in patients with lower extremity venous thrombosis: the patient was placed in a supine position on a catheter bed. The renal veins were examined by portable ultrasound to determine the left and right renal veins' position. The IVC diameter was measured (Figure 1) and was marked on the surface of the body.

The popliteal vein, femoral vein, ILIAC vein, bilateral renal vein, and IVC bifurcation were observed. After successful local anesthesia, the sendinger technique was used to insert the guidewire into the IVC via the femoral vein under portable ultrasound monitoring. The guidewire was fed into a conveyor (Figure 2). The guidewire was sent to the vena cava segment between the renal vein and the IVC, which confirmed the guidewire was in the IVC.

After the guidewire was fed into the $6 \mathrm{~F}$ sheath tube, the transmissible filter's tip was implanted into the renal 


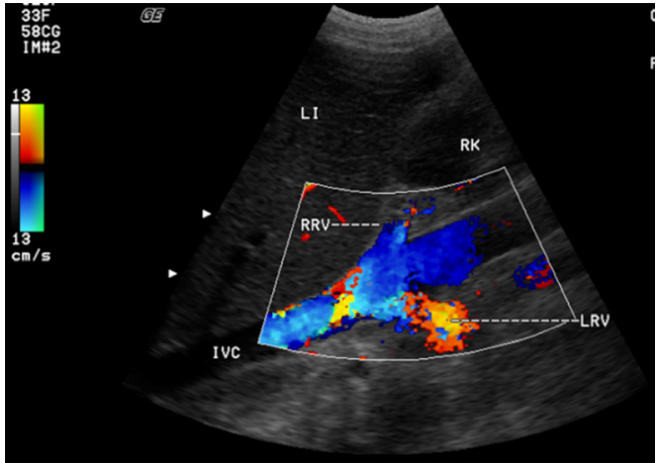

Figure 1 Ultrasound localization of the inferior vena cava and renal vein. IVC, inferior vena cava; RRV, right renal vein; LRV, left renal vein. RK, right kidney; LI, liver.

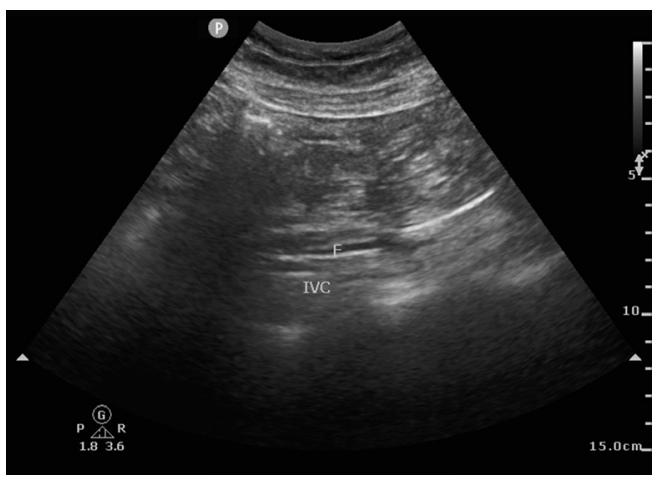

Figure 2 Ultrasound-guided wire development. IVC, inferior vena cava; F, filter.

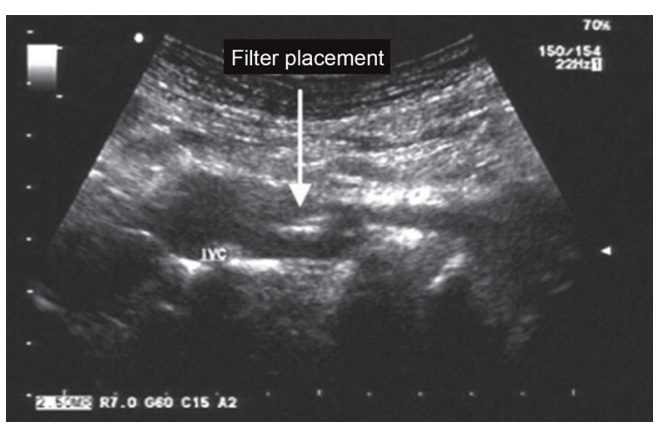

Figure 3 Filter placement. IVC, inferior vena cava.

vein's beginning under ultrasound guidance. The filter's location was determined by the development of the portable ultrasound filter (Figure 3). The filter was fixed 1-2 cm below the renal vein opening.

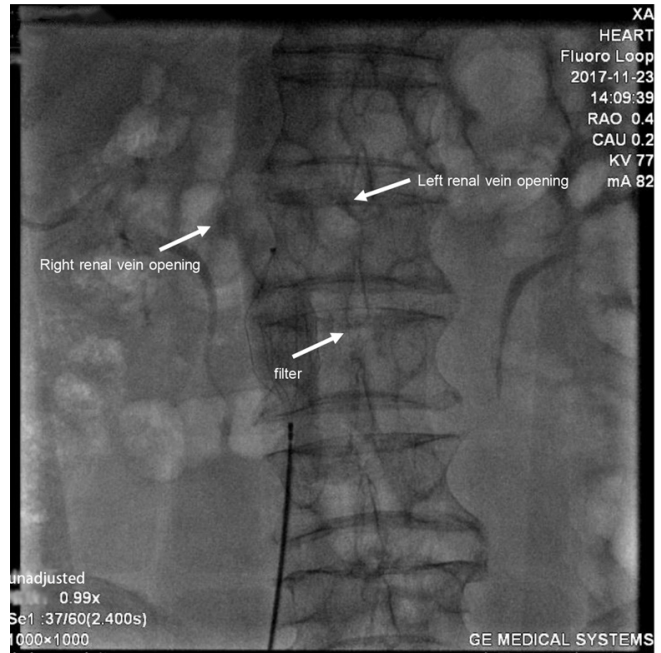

Figure 4 Release after DSA determines the filter position. DSA, digital subtraction angiography.

The renal vein opening, and filter implantation were reconfirmed by injection of contrast medium under the fluoroscope of DSA. If the filter is not placed in the predetermined position, the filter will be adjusted under the X-ray to re-adjust to the distal end of $1-2 \mathrm{~cm}$ below the renal vein's opening. After the filter position is determined again, the sheath tube is pushed back, and the filter is expanded by itself (Figure 4). Withdrawal of the sheath and delivery catheter and press the puncture point for 5-10 min.

\section{Statistical analysis}

\section{Statistical method}

All data were entered into the EPIDATA database by an expert. The statistical software SPSS 17.0 was used for statistical analysis. The mean \pm standard deviation expresses the measurement data $(\bar{x} \pm s)$. A paired sample $t$-test conducted the comparison within the group. An independent sample $t$-test was used for intergroup comparison. $\mathrm{P}<0.05$ was statistically significant.

\section{Sample size calculation}

The research group conducted basic research and preclinical research results, sample size calculation. At $\alpha=0.05$ level, to achieve $80 \%$ efficacy, 132 patients need to be enrolled in the study group. In this study, 200 eligible cases were included, and the corresponding efficacy was $95 \%$. 
Table 1 Comparison of general clinical data [n (\%)]

\begin{tabular}{|c|c|c|c|c|}
\hline Characteristic & Observation group & Control group & $\mathrm{t} / \chi^{2}$ & $\mathrm{P}$ \\
\hline Age (yeas) & $61.42 \pm 14.28$ & $62.68 \pm 14.82$ & -0.869 & 0.385 \\
\hline $\mathrm{BMI}\left(\mathrm{kg} / \mathrm{m}^{2}\right)$ & $21.40 \pm 1.95$ & $21.46 \pm 1.87$ & -0.314 & 0.754 \\
\hline Course of disease (d) & $1.96 \pm 0.13$ & $2.17 \pm 0.15$ & -1.563 & 0.119 \\
\hline
\end{tabular}

APTT, activated partial thromboplastin time.

Table 2 Comparison of operation conditions between two groups [n (\%)]

\begin{tabular}{|c|c|c|c|c|}
\hline Operation conditions & Observation group & Control group & $t / \chi^{2}$ & $\mathrm{P}$ \\
\hline Yes & $4(2.0)$ & $7(3.5)$ & & \\
\hline No & $120(60.0)$ & $116(58.0)$ & & \\
\hline $\mathrm{X}$-ray radiation, $\mathrm{n}(\%)$ & & & 400 & $<0.001$ \\
\hline No & $200(100.0)$ & 0 & & \\
\hline Contrast medium, $\mathrm{n}(\%)$ & & & 400 & $<0.001$ \\
\hline Yes & 0 & $200(100.0)$ & & \\
\hline No & $200(100.0)$ & 0 & & \\
\hline Pulmonary embolism, n (\%) & $6(3.0)$ & $7(3.5)$ & 0.080 & 0.778 \\
\hline Impairment of liver and kidney function, $\mathrm{n}(\%)$ & 0 & $11(5.5)$ & 11.311 & 0.001 \\
\hline
\end{tabular}

\section{Results}

\section{Comparison of general clinical data}

A total of 400 patients were studied, including 164 males $(41.0 \%)$ and 236 females (59.0\%). Average age: $62.05 \pm 14.29$. The average BMI was $21.43 \pm 1.45 \mathrm{~kg} / \mathrm{m}^{2}$. In the observation group, there were 200 patients, 80 men $(40.0 \%)$, the average age was $61.42 \pm 14.28$ years old. The control group consisted of 200 patients ( 84 males, $42.0 \%$ ), with an average age of $62.68 \pm 14.82$. There were no significant differences in sex, age, BMI, course of the disease, and Aptt between the two groups $(\mathrm{P}>0.05$, Table 1$)$.

\section{Comparison of operation conditions between two groups}

The results showed that the control group's operation time was significantly longer than that in the control group $(\mathrm{t}=-18.961, \mathrm{P}<0.001)$, and there is no $\mathrm{X}$-ray radiation and contrast medium injections in the observation group. There was no significant difference in puncture times and puncture site hematoma between the two groups $(\mathrm{P}>0.05)$. The results showed no significant difference in the postoperative complications between the two groups $\left(\chi^{2}=0.080, \mathrm{P}=0.778\right)$. The incidence of liver and kidney function damage in the control group was significantly greater than that in the Observation Group $\left(\chi^{2}=11.311, \mathrm{P}=0.001\right.$, Table 2$)$.

\section{Comparing the filter condition, blood vessel patency, and limb circumference difference between two groups at 1, 4, and $14 d$ after the operation}

The results showed that: 1, 4, 14 days after the operation, 
A

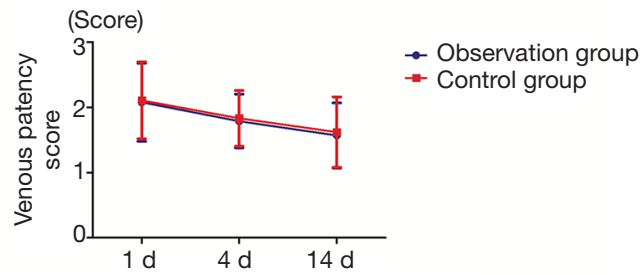

Comparison of venous patency scores at different time points

C

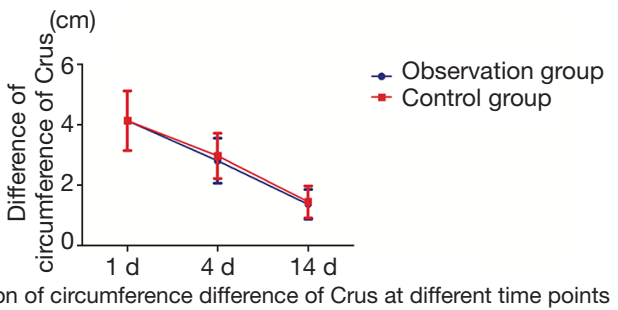

B

$(\mathrm{cm})$

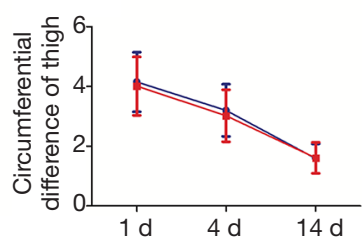

$\rightarrow$ Observation group

- Control group

Comparison of the difference of thigh circumference at different time points

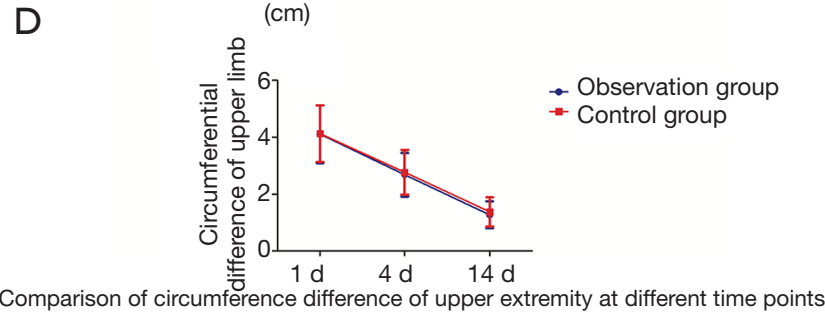

Figure 5 Comparison of various parameters at different time points. (A) Venous patency score; (B) thigh circumference difference; (C) crus circumference difference and (D) upper limb circumference difference.

A

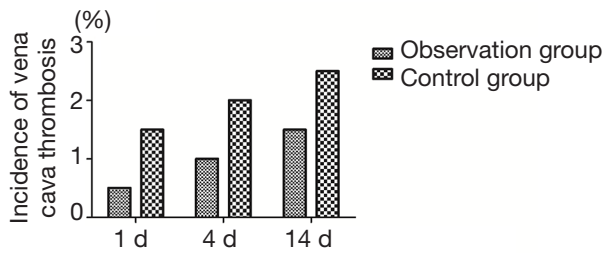

Comparison of the incidence of vena cava thrombosis at different time points

C

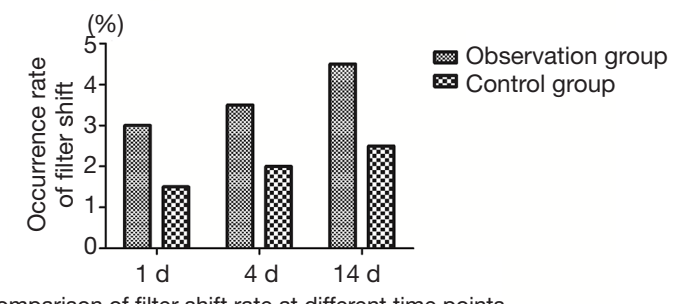

B

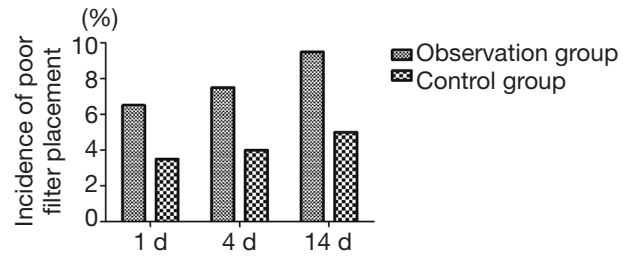

Comparison of the incidence of underposition of filters at different time points

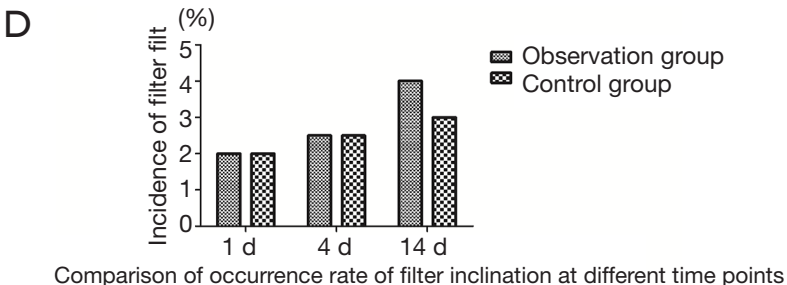

Figure 6 Comparison of the incidence of postoperative conditions at different time points. (A) Vena cava thrombosis, (B) underposition of filters, (C) filter shift and (D) filter inclination.

the filter condition, the degree of vascular patency, and the difference of peripheral diameter of venous embolism were compared between the two groups, and there were no significant differences between the two groups in the scores of venous patency, the proportion of vena cava thrombosis, the difference of limb circumferential diameter and the condition of filter $(\mathrm{P}>0.05)$. In each group, with time, the venous patency scores and the difference of limb circumference of venous embolism decrease difference was statistically significant $(\mathrm{P}<0.05)$.
There was no significant difference in the proportion of thrombus in vena cava and the filter condition between the two groups ( $\mathrm{P}>0.05$, Figures 5,6$)$.

\section{Comparison of Vascular Patency at 1, 2, 3, and 6 m after operation between two groups}

The vascular patency of $1,2,3$, and $6 \mathrm{~m}$ after the operation was compared between the two groups. The results showed that there was no significant difference between the two 


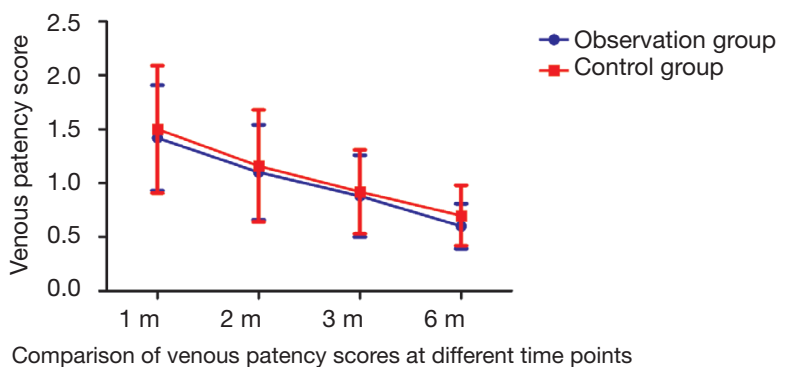

Figure 7 Comparison of venous patency scores at different time points.

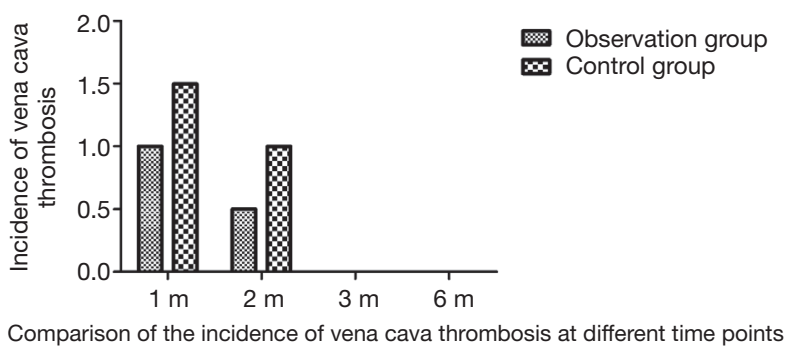

Figure 8 Comparison of the incidence of vena cava thrombosis at different time points.

groups $(\mathrm{P}>0.05)$. The pulse patency score of each group decreased with time. There were significant differences between the two groups except for 3 and $6 \mathrm{~m}(\mathrm{P}<0.05)$. There was no significant difference in the proportion of thrombus formation and the filter size between the two groups over time $(\mathrm{P}>0.05$, Figures 7,8$)$.

\section{Discussion}

DVT is one of the most common diseases after limb trauma. The incidence of venous thrombosis in the lower extremities after an earthquake is $11 \%$ (11). Cheng et al. (12) reviewed 352 cases of limb war wounds and 15 cases of death from 1968 to 2002 in the People's Liberation Army 303 hospital. The results showed that acute PE was the second cause of death after acute kidney failure, and $40-70 \%$ of PE occurred within 1 week after trauma (i.e., high-risk period of PE).

With the development of modernization and technology of weapons and equipment, severe limb battle injury is further increased. Acute PE, the most common DVT complication, has a high mortality rate $(13,14)$. The current clinical prevention of acute venous thrombosis of the limbs caused by PE methods includes drug prevention and mechanical prevention. Anticoagulant and thrombolytic drugs are the main drugs for drug prevention, but most war wounds of limbs are complicated with open injury, bleeding injury, or crush injury. Instrumental prophylaxis involves using a vena cava filter to block blood clots from entering the pulmonary artery and causing a fatal PE. The vena cava filter can significantly reduce the incidence of a PE caused by thrombus detachment. Rosenthal et al. (15) studied 94 patients with lower extremity venous thrombosis after multiple traumas. Using retrievable IVC filters significantly reduced the incidence of complications during hospitalization in critically ill patients, and only 1 patient (1\%) in the study had a complication of PE. Passman et al. (16) showed that the success rate of IVUS combined with transabdominal ultrasound was $97.7 \%$ (425/486 cases). Gilbert Aidinian et al. (17) have found that injuries to limbs from explosions and high-speed debris are common in the current war on terror. Lower limb thrombosis incidence after military trauma is significantly higher than the general population, and the condition is more complicated, often accompanied by complications including hemorrhagic shock, fracture, and kidney failure. All patients need repeated surgery and long-term rehabilitation treatment, seriously affecting the quality of life. For these patients, IVUS + ultrasound implantation's safety and effectiveness in the vena cava filter are recognized from early transportation inconvenience. Ganguli et al. (18) retrospectively compared 117 patients with IVUS filter implantation and 571 patients with X-ray filter implantation. The former's incidence of adverse reactions was $4.3 \%$ (4 cases with filter dislocation and 1 case with filter tilt). The latter's incidence was $0.6 \%$ ( 1 case of malposition and 1 case of inclination). After 463 days of follow-up, there was no difference in the incidence of complications between the two groups, so the ultrasound-guided filter implantation is feasible. However, IVUS equipment is too expensive, has not been widely used in China, the corresponding technology has fewer doctors, does not meet China's national conditions, and cannot be popularized in China. So, we try to use the portable ultrasound directly, under the guidance of ultrasound, to identify thrombus, renal vein, and IVC, implanted retrievable IVC filter, the same effect, and the cost is significantly reduced. Amankwah et al. (19) have studied the implantation of a biphasic ultrasound-guided filter through the abdomen, the success rate is higher, this large-scale ultrasound machine can guide the situation of 
the blood vessel and filter more highly, but the ultrasound machine occupies a larger area, affected by the hospital ward environment. Many hospitals in the country cannot achieve this, and portable ultrasound can be a satisfactory solution. Killingsworth et al. (20) performed a prospective study on 104 critically ill patients. The filter was implanted into the IVC by bedside ultrasound and intravascular contrast-enhanced ultrasound. The results showed that 3 patients had improper filter positioning, 2 patients had filtered inclination greater than 15, 1 patient had an arteriovenous fistula, and the 30-day mortality was $5.5 \%$. A single-center, 5-year follow-up study by the American College of Vascular Surgeons (21) showed 398 patients underwent bedside ultrasound + IVUS guided vena cava filter implantation, 262 of those who had anticoagulant contraindications, and 12 of whom developed perioperative complications including arteriovenous fistula, secondary thrombosis, local hematoma, filter tilt, and so on. The first 100 cases had 7\% operative complications, and the last 100 cases had only $2 \%$. In this study, only 1 patient had a poor filter position, which was slightly adjusted under X-ray. The reduction of complications was considered, which was related to the operator's skill and the complexity of the disease. In this study, only 1 patient with lower limb thrombosis was simple; no death occurred within 30 days, all patients were discharged. According to the randomized controlled trial, critical care patients have a $10 \%$ increased risk of thrombosis and $\mathrm{PE}$ even after routine anticoagulant therapy. The study of vena cava filter implantation is more important for critically ill patients, but ultrasound-guided filter implantation is still a blank in China.

$\mathrm{X}$-ray is a conventional and classical method to guide an IVC filter $(22,23)$. It has the advantages of accuracy and intuitionistic and is widely used in the clinic. However, this method has the following disadvantages: (I) the DSA machine's body is large and fixed, so it cannot be moved according to the need to apply to the first-aid places; (II) DSA machines are expensive. Even though only a few of them are available in top hospitals, they cannot meet the needs of large-scale Mass Limb Warfare Trauma; (III) DSA requires a high-intensity continuous AC power supply, limited in the first-aid places. Most of the limb battle trauma is associated with crush injury or acute renal injury caused by insufficient perfusion of blood. The iodine contrast medium is contraindicated.

To sum up, portable ultrasound has the following advantages: (I) small volume, lightweight, flexible and convenient use can be operated in a single palm, according to the need to move its position in the first aid sites; (II) self-contained rechargeable battery, no external power supply, not affected by the power of advanced first-aid sites; (III) cheap, only $1 / 20$ of the price of DSA machine; (IV) it should be wide range: ultrasound-guided vena cava filter implantation without contraindication can be applied to the patients with contraindication of $\mathrm{X}$-ray guidance and unsuitable for $\mathrm{X}$-ray guidance, including the patients with contrast agent allergy, renal insufficiency, and severe disease.

This study is the first to study the feasibility of portable ultrasound-guided retrievable vena cava filter implantation to prevent acute $\mathrm{PE}$ after limb trauma and the prevention of acute PE after limb trauma. It supplies a scientific and effective intervention method and clinical data to prevent $\mathrm{PE}$ under simple conditions, including the front-line firstaid sites. The portable ultrasound-guided retrievable vena cava filter implantation can be used in battlefield first aid, earthquake, and disaster rescue, reducing the incidence of a PE caused by high energy injury and improving patients' survival rate after limb trauma.

This study has some limitations; the sample size is limited, whether it can be extended in clinical need of a larger scale, more disease research. Secondly, the subjects selected in this study are non-obese people. A study by Qin (24) showed that the renal vein's transabdominal ultrasound might be influenced by abdominal gas, obesity, vascular malformation, etc. However, studies by Liu et al. (25) have shown obesity has no significant effect on ultrasound-guided IVC implantation. There is a similar problem with portable ultrasound; whether the ultrasound-guided retrievable vena cava filter implantation in patients with flatulence, abdominal wall edema, obesity, and vascular malformation has a significant deviation from its position needs further study.

The success rate of portable ultrasound-guided retrievable vena cava filter implantation was like that of $\mathrm{X}$-ray-guided implantation, but there was no significant difference. It is proved the portable ultrasound-guided retrievable Vena cava filter implantation is safe and feasible to prevent acute $\mathrm{PE}$ after battle injury of limbs.

\section{Acknowledgments}

Funding: This study was supported by the Capital Clinical Application Research Project (No. Z181100001718042).

\section{Footnote}

Reporting Checklist: The authors have completed the 
STROBE reporting checklist. Available at http://dx.doi. org/10.21037/atm-21-1290

Data Sharing Statement: Available at http://dx.doi. org/10.21037/atm-21-1290

Conflicts of Interest: All authors have completed the ICMJE uniform disclosure form (available at http://dx.doi. org/10.21037/atm-21-1290). The authors have no conflicts of interest to declare.

Ethical Statement: The authors are accountable for all aspects of the work in ensuring that questions related to the accuracy or integrity of any part of the work are appropriately investigated and resolved. All procedures performed in this study involving human participants were in accordance with the Declaration of Helsinki (as revised in 2013). The study was approved by Ethics Committee of Chinese PLA General Hospital and informed consent was taken from all the patients.

Open Access Statement: This is an Open Access article distributed in accordance with the Creative Commons Attribution-NonCommercial-NoDerivs 4.0 International License (CC BY-NC-ND 4.0), which permits the noncommercial replication and distribution of the article with the strict proviso that no changes or edits are made and the original work is properly cited (including links to both the formal publication through the relevant DOI and the license). See: https://creativecommons.org/licenses/by-nc-nd/4.0/.

\section{References}

1. Nantulya VM, Sleet DA, Reich MR, et al. Introduction: the global challenge of road traffic injuries: can we achieve equity in safety? Inj Control Saf Promot 2003;10:3-7.

2. Varghese M, Mohan D. Transportation injuries in rural Haryana, North India. Proceedings of the International Conference on Traffic Safety 2003:326-9.

3. Cannon KA, Badiee J, Wallace JD, et al. The prevalence of chronic deep venous thrombosis in trauma: Implications for hospitals and patients. J Trauma Acute Care Surg 2018;84:170-4.

4. Sørensen HT, Horvath-Puho E, Lash TL, et al. Heart disease may be a risk factor for pulmonary embolism without peripheral deep venous thrombosis. Circulation 2011;124:1435-41.

5. O'Malley KF, Ross SE. Pulmonary embolism in major trauma patients. J Trauma 1990;30:748-50.

6. Ghaye B, Willems V, Nchimi A, et al. Relationship between the extent of deep venous thrombosis and the extent of acute pulmonary embolism as assessed by CT angiography. Br J Radiol 2009;82:198-203.

7. Shamian B, Chamberlain RS. The role for prophylaxis inferior vena cava filters in patients undergoing bariatric surgery: replacing anecdote with evidence. Am Surg 2012;78:1349-61.

8. Stearley HE. Patients' outcomes: intrahospital transportation and monitoring of critically ill patients by a specially trained ICU nursing staff. Am J Crit Care 1998;7:282-7.

9. Sato DT, Robinson KD, Gregory RT, et al. Duplex directed caval filter insertion in multi-trauma and critically ill patients. Ann Vasc Surg 1999;13:365-71.

10. Conners MS 3rd, Becker S, Guzman RJ, et al. Duplex scan-directed placement of inferior vena cava filters: a fiveyear institutional experience. J Vasc Surg 2002;35:286-91.

11. Mingfu D. Screening, Prevention and Treatment of Venous Thrombosis for 235 Lushan Earthquake Victims. Chinese Journal of Evidence-based Medicine 2013.

12. Cheng CZ, Zhao DH, Li QY, Qu HY, Chen BC, Lin ZD. Logistic regression analysis of the risk factors of acute renal failure complicating limb war injuries. Medical Journal of Chinese People's Liberation Army 2011;36:671 (Chinese: http://www.cnki.com.cn/article/cjfdtotaljfjy201106039.htm).

13. Geerts WH, Code KI, Jay RM, et al. A prospective study of venous thromboembolism after major trauma. $\mathrm{N}$ Engl J Med 1994;331:1601-6.

14. Arnold DM, Kahn SR, Shrier I. Missed opportunities for prevention of venous thromboembolism: an evaluation of the use of thromboprophylaxis guidelines. Chest 2001;120:1964-71.

15. Rosenthal D, Wellons ED, Levitt AB, et al. Role of prophylactic temporary inferior vena cava filters placed at the ICU bedside under intravascular ultrasound guidance in patients with multiple trauma. J Vasc Surg 2004;40:958-64.

16. Passman MA, Dattilo JB, Guzman RJ, et al. Bedside placement of inferior vena cava filters by using transabdominal duplex ultrasonography and intravascular ultrasound imaging. J Vasc Surg 2005;42:1027-32.

17. Aidinian G, Fox CJ, White PW, et al. Intravascular ultrasound--guided inferior vena cava filter placement in the military multitrauma patients: a single-center experience. Vasc Endovascular Surg 2009;43:497-501. 
18. Ganguli S, Hawkins BM, Abtahian F, et al. Comparison of Inferior Vena Cava Filters Placed at the Bedside via Intravenous Ultrasound Guidance Versus Fluoroscopic Guidance. Ann Vasc Surg 2017;39:250-5.

19. Amankwah KS, Seymour K, Costanza M, et al. Transabdominal duplex ultrasonography for bedside inferior vena cava filter placement: examples, technique, and review. Vasc Endovascular Surg 2009;43:379-84.

20. Killingsworth CD, Taylor SM, Patterson MA, et al. Prospective implementation of an algorithm for bedside intravascular ultrasound-guided filter placement in critically ill patients. J Vasc Surg 2010;51:1215-21.

21. Glocker RJ, Awonuga O, Novak Z, et al. Bedside inferior vena cava filter placement by intravascular ultrasound in critically ill patients is safe and effective for an extended time. J Vasc Surg Venous Lymphat Disord 2014;2:377-82.

Cite this article as: Zhu H, Du WJ, Wang XH, Yang Y, Dekyi, Chen YD, Zhao J. Feasibility study of hand-carried ultrasoundguided retrievable inferior vena cava filter placement. Ann Transl Med 2021;9(8):689. doi: 10.21037/atm-21-1290
22. Cheng ZY, Peng XX, Zhang YW. Inferior vena cava filter placement in the prevention of pulmonary embolism and its complications. Chinese Journal of Interventional Imaging and Therapy 2007;4:19-22.

23. Xu K, Zhou YB, Wang AL, et al. Clinical study of domestic ZQL-type retrievable vena cava filter. Chinese Medical Journal 2009;122:140-4.

24. Qin X, Lu C, Ren P, et al. New method for ultrasoundguided inferior vena cava filter placement. J Vasc Surg Venous Lymphat Disord 2018;6:450-6.

25. Liu Y, Zhou H, Chen C, et al. Assessment of the safety and efficacy of bedside ultrasound guidance for inferior vena cava filter placement in critically ill intensive care unit patients. Ultrasound Med Biol 2015;41:929-35.

(English Language Editor: J. Chapnick) 